\title{
Three-Field Modelling of Nonlinear Nonsmooth Boundary Value Problems and Stability of Differential Mixed Variational Inequalities
}

\author{
J. Gwinner \\ Institut für Mathematik und Rechneranwendung, Universität der Bundeswehr München, 85577 Neubiberg/München, Germany \\ Correspondence should be addressed to J. Gwinner; joachim.gwinner@unibw.de
}

Received 29 December 2012; Accepted 15 March 2013

Academic Editor: Donal O’Regan

Copyright (C) 2013 J. Gwinner. This is an open access article distributed under the Creative Commons Attribution License, which permits unrestricted use, distribution, and reproduction in any medium, provided the original work is properly cited.

\begin{abstract}
The purpose of this paper is twofold. Firstly we consider nonlinear nonsmooth elliptic boundary value problems, and also related parabolic initial boundary value problems that model in a simplified way steady-state unilateral contact with Tresca friction in solid mechanics, respectively, stem from nonlinear transient heat conduction with unilateral boundary conditions. Here a recent duality approach, that augments the classical Babuška-Brezzi saddle point formulation for mixed variational problems to twofold saddle point formulations, is extended to the nonsmooth problems under consideration. This approach leads to variational inequalities of mixed form for three coupled fields as unknowns and to related differential mixed variational inequalities in the time-dependent case. Secondly we are concerned with the stability of the solution set of a general class of differential mixed variational inequalities. Here we present a novel upper set convergence result with respect to perturbations in the data, including perturbations of the associated nonlinear maps, the nonsmooth convex functionals, and the convex constraint set. We employ epiconvergence for the convergence of the functionals and Mosco convergence for set convergence. We impose weak convergence assumptions on the perturbed maps using the monotonicity method of Browder and Minty.
\end{abstract}

\section{Introduction}

The classical Babuška-Brezzi theory for mixed variational problems has been extended by Gatica $[1,2]$ to some classes of variational problems and nonlinear operator equations. This extension leads to three-field variational models that can be understood as dual-dual mixed variational models or as twofold saddle point formulations. Such augmented variational models are well adapted for multiphysics problems with different coupled unknown quantities and in particular for engineering problems, where speaking in terms of solid mechanics, strains and stresses are often of more interest then the displacements.

In a series of papers Gatica with coauthors applied his duality approach to the numerical treatment of various linear/nonlinear interior/exterior elliptic boundary value problems by the finite element method (FEM), the boundary element method (BEM), or by the coupling of these discretization methods. Here we refer to the paper of Gatica et al. [3] that presents a numerical analysis of nonlinear two-fold saddle point problems involving a nonlinear operator equation with a uniformly monotone operator.

This novel duality approach to nonlinear nonsmooth boundary value problems has to be distinguished from the standard duality approach which hinges on the Lagrange duality theory of convex analysis in calculus of variations (see [4] for a systematic study) and which is employed in the numerical FEM analysis of various unilateral boundary and obstacle problems as pioneered by Haslinger and Lovišek $[5,6]$; see also the monograph [7].

In this paper, we address a simplified scalar model of steady-state unilateral contact problems with Tresca friction and nonlinear transient heat conduction problems with unilateral boundary conditions. We extend the duality approach of Gatica to such problems. This approach leads to variational inequalities of mixed form for three coupled fields as unknowns and to related differential mixed variational inequalities (DMVI) in the time-dependent case. 
Differential variational inequalities have recently been introduced and studied in depth by Pang and Stewart [8] in finite dimensions as a new modeling paradigm of variational analysis. In their seminal paper the authors have already shown that this new class of differential inclusions contains ordinary differential equations with possibly discontinuous right-hand sides, differential algebraic systems, dynamic complementarity systems, and evolutionary variational systems. More recently, some results of [8] have been extended to DMVI by Li et al. [9] in finite dimensions.

Furthermore in this paper, we are concerned with stability of the solution set to DMVI. In this connection, let us refer to [10], where a Lyapunov approach is developed for strong solutions of evolution variational inequalities and to [11], where first several sensitivity results are established for initial value problems of ordinary differential equations with nonsmooth right hand sides and then applied to treat differential variational inequalities. Related stability results for more general evolution inclusions by Papageorgiou [12, 13] and by $\mathrm{Hu}$ and Papageorgiou in the memoir [14] are not applicable here since these results are limited to finite dimensions, respectively, and need more stringent compactness assumptions.

Here we present a novel upper set convergence result for DMVI with respect to perturbations in the data. In particular, we admit perturbations of the nonlinear maps, of the nonsmooth convex functionals, and the convex constraint set that describe the DMVI. We employ epiconvergence for the convergence of the functionals and Mosco convergence for set convergence. Since our analysis of the underlying mixed nonlinear variational inequality relies on the monotonicity method of Browder and Minty (see e.g., $[15,16]$ ), we need to impose comparably weak convergence assumptions on the perturbations in the nonlinear maps. Thus we extend the stability results of [17] (without considering slow solutions here) and of [18] to this new more general class of mixed differential variational inequalities.

The outline of this paper is as follows. In Sections 2 and 3, we show how the duality approach of Gatica can be extended to a nonlinear transient heat problem with unilateral boundary conditions and to a scalar nonsmooth boundary value problem modelling the steady-state unilateral contact of an elastic body with Tresca friction. Thus we obtain variational inequalities of mixed form involving three unknown fields and related differential mixed variational inequalities (DMVI) in the time-dependent case. Then in Section 4, we turn to the stability analysis of a general class of DMVI. After a discussion of some preliminaries including Mosco set convergence and epiconvergence (Section 4.2), we establish our novel stability result in Section 4.3 based on the Browder-Minty monotonicity method. Section 5 gives an outlook to some open directions of research.

\section{A Nonlinear Nonsmooth Boundary Value Problem from Heat Conduction}

In this section we consider a nonlinear boundary value problem with Signorini boundary conditions that arises from nonlinear heat conduction [19] with semipermeable walls [20]. We first show how the steady-state problem can be variationally formulated as a variational inequality in mixed form. Then we turn to the transient problem and derive the associated differential mixed variational inequality.

To describe the problem of interest, let $\Omega$ be a bounded simply connected domain in $\mathbb{R}^{2}$ with the Lipschitz boundary $\partial \Omega=\Gamma[21]$. Then $\boldsymbol{v}$, the outward normal to $\Gamma$, exists almost everywhere and $\boldsymbol{v} \in\left[L^{\infty}(\Gamma)\right]^{2}$ (see $\left[21\right.$, Lemma 2.4.2]). Let $\Gamma_{D}$ and $\Gamma_{S}$ be parts of $\Gamma$, such that $\left|\Gamma_{D}\right|>0,\left|\Gamma_{S}\right|>0, \Gamma=\overline{\Gamma_{D}} \cup \overline{\Gamma_{S}}$ and $\Gamma_{D} \cap \Gamma_{S}=\emptyset$. Also let $a_{i}: \Omega \times \mathbb{R}^{2} \rightarrow \mathbb{R}, i=1,2$ be nonlinear functions satisfying certain conditions (specified in what follows), and write

$$
\mathbf{a}(x, \xi):=\left(a_{1}(x, \xi), a_{2}(x, \xi)\right)^{T} \quad \forall(x, \xi) \in \Omega \times \mathbb{R}^{2} .
$$

Then, for a given right-hand side $f: \Omega \rightarrow \mathbb{R}$, given function $g: \partial \Omega \rightarrow \mathbb{R}$ defining the boundary conditions, we look for a function $u: \bar{\Omega} \rightarrow \mathbb{R}$, such that

$$
\begin{gathered}
-\operatorname{div} \mathbf{a}(\cdot, \nabla u(\cdot))=f \quad \text { on } \Omega, \\
u=g \quad \text { on } \Gamma_{D}, \\
u \geq g, \quad \mathbf{a}(\cdot, \nabla u(\cdot)) \cdot v \geq 0, \quad(u-g) \mathbf{a}(\cdot, \nabla u(\cdot)) \cdot \boldsymbol{v}=0, \\
\text { on } \Gamma_{S},
\end{gathered}
$$

where div is the usual divergence operator. We introduce the gradient $\mathbf{p}:=\nabla u$ in $\Omega$ and the flux $\sigma:=\mathbf{a}(\cdot, \mathbf{p})$ in $\Omega$ as additional unknowns. In this way, the elliptic pde (2) in (2) writes as the three equations

$$
\begin{gathered}
-\operatorname{div} \boldsymbol{\sigma}=f, \\
\mathbf{a}(\cdot, \mathbf{p})=\boldsymbol{\sigma}, \\
\mathbf{p}=\nabla u,
\end{gathered}
$$

that should hold in the distributional sense in $\Omega$. By this reformation we can relax the regularity of the unknown $u$. We require that $u \in L^{2}(\Omega), \mathbf{p} \in L^{2}\left(\Omega, \mathbb{R}^{2}\right)$, and $\boldsymbol{\sigma} \in H(\operatorname{div}, \Omega):=$ $\left\{\boldsymbol{\sigma} \in L^{2}\left(\Omega, \mathbb{R}^{2}\right) \mid \operatorname{div} \boldsymbol{\sigma} \in L^{2}(\Omega)\right\}$. Thus testing (3) $)_{1},(3)_{2}$ with $v \in L^{2}(\Omega), \mathbf{q} \in L^{2}\left(\Omega, \mathbb{R}^{2}\right)$, respectively, gives

$$
\begin{gathered}
-\int_{\Omega} \operatorname{div} \boldsymbol{\sigma} v d \mathbf{x}=\int_{\Omega} f v d \mathbf{x}=:(f, v)_{L^{2}(\Omega)}, \\
\int_{\Omega} \mathbf{a}(\cdot, \mathbf{p}) \cdot \mathbf{q} d \mathbf{x}=\int_{\Omega} \boldsymbol{\sigma} \cdot \mathbf{q} d \mathbf{x}=:(\boldsymbol{\sigma}, \mathbf{q})_{L^{2}\left(\Omega, \mathbb{R}^{2}\right)}
\end{gathered}
$$

For the last equation $(3)_{3}$ in (3) we incorporate the boundary conditions and use Green's formula (see [22])

$$
(\nabla \varphi, \boldsymbol{\tau})_{L^{2}\left(\Omega, \mathbb{R}^{2}\right)}+(\varphi, \operatorname{div} \boldsymbol{\tau})_{L^{2}(\Omega)}=\left\langle\gamma_{0} \varphi, \gamma_{\nu} \boldsymbol{\tau}\right\rangle_{L^{2}(\Gamma)}
$$

for $\varphi \in H^{1}(\Omega), \boldsymbol{\tau} \in H(\operatorname{div}, \Omega)$, where $\gamma_{0} \varphi$ and $\gamma_{\nu} \boldsymbol{\tau}=\boldsymbol{\tau} \cdot \boldsymbol{\nu}$ denote the traces. Testing $(3)_{3}$ with $\boldsymbol{\tau}-\boldsymbol{\sigma} \in H(\operatorname{div}, \Omega)$ results in

$$
\begin{aligned}
& (\mathbf{p}, \boldsymbol{\tau}-\boldsymbol{\sigma})_{L^{2}\left(\Omega, \mathbb{R}^{2}\right)}+(u, \operatorname{div} \boldsymbol{\tau}-\operatorname{div} \boldsymbol{\sigma})_{L^{2}(\Omega)} \\
& =\left\langle\gamma_{0} u, \gamma_{\nu}(\boldsymbol{\tau}-\boldsymbol{\sigma})\right\rangle \\
& =\left\langle\gamma_{0} u-g, \gamma_{\nu} \boldsymbol{\tau}\right\rangle-\left\langle\gamma_{0} u-g, \gamma_{\nu} \boldsymbol{\sigma}\right\rangle+\left\langle g, \gamma_{\nu}(\boldsymbol{\tau}-\boldsymbol{\sigma})\right\rangle .
\end{aligned}
$$


By the boundary conditions, the previous second term vanishes. The second inequality in the Signorini boundary condition $(2)_{3}$ tells us that we have to require that $\boldsymbol{\sigma}$ belongs to the convex cone as follows:

$$
\begin{aligned}
H_{+} & :=H_{+}\left(\operatorname{div}, \Omega, \Gamma_{S}\right) \\
& :=\left\{\boldsymbol{\tau} \in H(\operatorname{div}, \Omega): \gamma_{\nu} \boldsymbol{\tau} \mid \Gamma_{S} \geq 0\right\},
\end{aligned}
$$

where " $\geq 0$ " means that $\left\langle\gamma_{0} \varrho, \gamma_{\nu} \boldsymbol{\tau}\right\rangle \geq 0$ for any smooth function $\varrho$ on $\bar{\Omega}$ with $\varrho=0$ on $\Gamma_{D}$ and $\varrho \geq 0$ on $\Gamma_{S}$. Thus we obtain for any $\tau \in H_{+}\left(\operatorname{div}, \Omega, \Gamma_{S}\right)$,

$$
\begin{gathered}
(\mathbf{p}, \boldsymbol{\tau}-\boldsymbol{\sigma})_{L^{2}\left(\Omega, \mathbb{R}^{2}\right)}+(u, \operatorname{div}(\boldsymbol{\tau}-\boldsymbol{\sigma}))_{L^{2}(\Omega)} \\
\geq\left\langle g, \gamma_{\nu}(\boldsymbol{\tau}-\boldsymbol{\sigma})\right\rangle_{L^{2}\left(\Gamma_{S}\right)} .
\end{gathered}
$$

Altogether we arrive at the following variational inequality of mixed form: find $[\mathbf{p}, \boldsymbol{\sigma}, u] \in L^{2}\left(\Omega, \mathbb{R}^{2}\right) \times H_{+}\left(\operatorname{div}, \Omega, \Gamma_{S}\right) \times$ $L^{2}(\Omega)$, such that for all $[\mathbf{q}, \boldsymbol{\tau}, v] \in L^{2}\left(\Omega, \mathbb{R}^{2}\right) \times H_{+}\left(\operatorname{div}, \Omega, \Gamma_{S}\right) \times$ $L^{2}(\Omega)$,

$$
\begin{gathered}
(\mathbf{a}(\cdot, \mathbf{p}), \mathbf{q})-(\boldsymbol{\sigma}, \mathbf{q})=0, \\
(\mathbf{p}, \boldsymbol{\tau}-\boldsymbol{\sigma})+(u, \operatorname{div},(\boldsymbol{\tau}-\boldsymbol{\sigma})) \geq\left\langle g, \gamma_{\nu}(\boldsymbol{\tau}-\boldsymbol{\sigma})\right\rangle_{\Gamma_{S}}, \\
-(v, \operatorname{div} \boldsymbol{\sigma})=(f, v) .
\end{gathered}
$$

Note that if the functions $a_{i}$ are Caratheodory functions and satisfy the growth conditions: $\exists C>0, \varphi_{i} \in L^{2}(\Omega), i=$ 1,2 such that

$$
\left|a_{i}(x, \xi)\right| \leq C\{1+|\xi|\}+\left|\varphi_{i}(x)\right|, \quad \forall \xi \in \mathbb{R}^{2} \text {, a.e. } x \in \Omega,
$$

then we obtain the Nemytskii operator $\mathbf{q} \in L^{2}\left(\Omega, \mathbb{R}^{2}\right) \mapsto$ $\mathbf{a}(\cdot, \mathbf{q}) \in L^{2}\left(\Omega, \mathbb{R}^{2}\right)$. Clearly, this nonlinear operator inherits monotonicity from the vector field $\mathbf{a}$; see, for example, [15].

Now we turn to the time-dependent case. Let $I=(0, T)$ be the given time interval. Then instead of the elliptic pde (2) we have the parabolic pde

$$
\partial_{t} u-\operatorname{div} \mathbf{a}(\cdot, \nabla u(\cdot))=f \quad \text { in } \Omega \times I .
$$

Also the boundary conditions have to hold in $\Omega \times I$, where we assume the gap function $g$ to be time-independent. In addition, we have the initial condition $u=u_{0}$ in $\Omega \times\{0\}$ with some given $u_{0}$.

As the solution space for the unknown $u$, we introduce

$$
W:=\left\{u \in L^{2}(\Omega \times I): \partial_{t} u \in L^{2}(\Omega \times I)\right\}
$$

Then the three-field variational formulation of the considered initial boundary value problem reads as the following differential mixed variational inequality: find $[u, \mathbf{p}, \boldsymbol{\sigma}] \in W \times$
$L^{2}\left(\Omega \times I, \mathbb{R}^{2}\right) \times L^{2}\left(I, H_{+}\left(\operatorname{div}, \Omega, \Gamma_{S}\right)\right)=: \mathscr{X}$, such that for all $[v, \mathbf{q}, \boldsymbol{\tau}] \in \mathscr{X}$ there holds

$$
\begin{gathered}
\int_{I} \int_{\Omega}\left(\partial_{t} u-\operatorname{div} \boldsymbol{\sigma}\right) v d x d t=\int_{I} \int_{\Omega} f v d x d t, \\
\int_{I} \int_{\Omega} \mathbf{a}(\cdot, \mathbf{p}) \cdot \mathbf{q} d x d t-\int_{I} \int_{\Omega} \boldsymbol{\sigma} \cdot \mathbf{q} d x d t=0, \\
\int_{I} \int_{\Omega} \mathbf{p} \cdot(\boldsymbol{\tau}-\boldsymbol{\sigma}) d x d t+\int_{I} \int_{\Omega} u \operatorname{div}(\boldsymbol{\tau}-\boldsymbol{\sigma}) d x d t \\
\geq \int_{I} \int_{\Gamma_{S}} g(\boldsymbol{\tau}-\boldsymbol{\sigma}) \cdot \boldsymbol{v} d s d t .
\end{gathered}
$$

\section{A Simplified Scalar Nonsmooth Boundary Value Problem from Frictional Contact}

In this section we treat a non-smooth boundary value problem that can be considered as a simplified scalar model of a nonsmooth contact mechanics problem involving Tresca friction and a unilateral constraint of an elastic body with a rigid foundation. Instead of the vector Navier-Lamé pde system or a nonlinear extension of it to model nonlinear elastic material, we are concerned with a nonlinear Helmholtzlike pde. This pde will be complemented by nonclassical boundary conditions involving the non-smooth modulus function. We show how a three-field modelling transforms this nonsmooth boundary value problem to a variational inequality of mixed type.

Similar to the preceding section, let $\Omega \subset \mathbb{R}^{2}$ be a bounded plane domain with the Lipschitz boundary. Here instead of the Navier-Lamé-system and instead of (2) we treat the elliptic Helmholz-like partial differential equation

$$
-\operatorname{div} \mathbf{a}(\cdot, \nabla u(\cdot))+u=f \quad \text { in } \Omega .
$$

Again we use the gradient $\mathbf{p}:=\nabla u$ in $\Omega$ and the flux $\boldsymbol{\sigma}:=$ $\mathbf{a}(\cdot, \mathbf{p})$ in $\Omega$ as additional unknowns. In this way, the elliptic pde (14) writes as the following three equations:

$$
\begin{gathered}
-\operatorname{div} \boldsymbol{\sigma}+u=f, \\
\mathbf{a}(\cdot, \mathbf{p})=\boldsymbol{\sigma}, \\
\mathbf{p}=\nabla u,
\end{gathered}
$$

that should hold in the distributional sense in $\Omega$. By this reformation we can again relax the regularity of the unknown $u$. We require that $u \in L^{2}(\Omega), \mathbf{p} \in L^{2}\left(\Omega, \mathbb{R}^{2}\right)$, and $\boldsymbol{\sigma} \in$ $H(\operatorname{div}, \Omega)$. Thus testing $(15)_{1}$, (15) 2 with $v \in L^{2}(\Omega), \mathbf{q} \in$ $L^{2}\left(\Omega, \mathbb{R}^{2}\right)$, respectively, gives

$$
\begin{gathered}
-\int_{\Omega} \operatorname{div} \boldsymbol{\sigma} v+u v d \mathbf{x}=\int_{\Omega} f v d \mathbf{x}=(f, v)_{L^{2}(\Omega)}, \\
\int_{\Omega} \mathbf{a}(\cdot, \mathbf{p}) \cdot \mathbf{q} d \mathbf{x}=\int_{\Omega} \boldsymbol{\sigma} \cdot \mathbf{q} d \mathbf{x}=(\boldsymbol{\sigma}, \mathbf{q})_{L^{2}\left(\Omega, \mathbb{R}^{2}\right)} .
\end{gathered}
$$


Now with the given function $g \in L^{\infty}(\Gamma)$ with $g(x) \geq$ $g_{0}>0$ a.e. on $\Gamma$, we impose the nonclassical boundary conditions

$$
\begin{gathered}
|u| \leq g, \\
u(\sigma \cdot \nu)=-g|\sigma \cdot \nu| .
\end{gathered}
$$

Note that (17) is equivalent to

$$
\begin{gathered}
|u|<g \Longrightarrow \boldsymbol{\sigma} \cdot \boldsymbol{\nu}=0, \\
|u|=g \Longrightarrow \boldsymbol{\sigma} \cdot \boldsymbol{\nu}=-\lambda u \quad \text { for some } \lambda \geq 0 .
\end{gathered}
$$

These implications reflect Tresca's law of friction (given friction model) and the more general Coulomb's law of friction [20]. Namely, with $\boldsymbol{\sigma} \cdot \boldsymbol{\nu}$ denoting (the tangential component of) the traction in the general elasticity problem, the first implication means that when the body is not in contact with the obstacle, there is no tangential stress due to friction. If on the other hand, the body is in contact with the rigid obstacle, then-this is the meaning of the second implication - there arises a tangential stress proportional and opposite to the tangential displacement $u$.

To obtain a variational formulation of the boundary condition (17), we consider the boundary integral

$$
\left\langle\gamma_{0} u, \gamma_{\nu}(\boldsymbol{\tau}-\boldsymbol{\sigma})\right\rangle+\int_{\Gamma} g\{|\boldsymbol{\tau} \cdot \boldsymbol{\nu}|-|\boldsymbol{\sigma} \cdot \boldsymbol{\nu}|\} d s
$$

and claim that this boundary integral is nonnegative for any $\tau \in H(\operatorname{div}, \Omega)$.

Indeed, if $|u|<g$ holds on some part of $\Gamma$, then $\gamma_{\nu} \sigma=$ $\boldsymbol{\sigma} \cdot \boldsymbol{\nu}=0$, and the integrand reduces to

$$
\gamma_{0} u \gamma_{\nu} \tau+g\left|\gamma_{\nu} \tau\right| \geq-\left|\gamma_{0} u\right|\left|\gamma_{\nu} \tau\right|+g\left|\gamma_{\nu} \tau\right| \geq 0
$$

If otherwise $|u|=g$ holds, then insert $\gamma_{\nu} \sigma=-\lambda \gamma_{0} u$, and the integrand becomes

$$
\begin{gathered}
\gamma_{0} u \gamma_{\nu} \tau+\lambda\left|\gamma_{0} u\right|^{2}+g\left|\gamma_{\nu} \tau\right|-g \lambda\left|\gamma_{0} u\right| \\
=\gamma_{0} u \gamma_{\nu} \tau+g\left|\gamma_{\nu} \tau\right| \\
\geq-g\left|\gamma_{\nu} \tau\right|+g\left|\gamma_{\nu} \tau\right|=0 .
\end{gathered}
$$

Thus we are led to the convex (actually sublinear) nonsmooth functional

$$
j(\boldsymbol{\tau}):=\int_{\Gamma} g|\boldsymbol{\tau} \cdot \boldsymbol{\nu}| d s .
$$

Remark 1. The obtained convex functional $j$ and the constraint $|u| \leq g$ are related by Fenchel duality of convex analysis, see for example [4]. Namely, introduce the convex, lower semicontinuous, proper function $\varphi(y):=g|y|$ with $g>0$. Then the Fenchel dual function

$$
\varphi^{*}(z):=\sup _{y}\{\langle y, z\rangle-\varphi(y)\}
$$

becomes

$$
\varphi^{*}(z)= \begin{cases}0 & \text { if }|z| \leq g \\ \infty & \text { otherwise }\end{cases}
$$

which is the indicator function of the previous constraint. Moreover it is known that the Fenchel bidual function $\left(\varphi^{*}\right)^{*}$ coincides with $\varphi$.

Finally in virtue of Green's formula (5), the proven claim provides the variational inequality of the second kind as follows:

$$
\begin{aligned}
& (\mathbf{p}, \boldsymbol{\tau}-\boldsymbol{\sigma})_{L^{2}\left(\Omega, \mathbb{R}^{2}\right)}+(u, \operatorname{div}(\boldsymbol{\tau}-\boldsymbol{\sigma}))_{L^{2}(\Omega)} \\
& \quad+j(\boldsymbol{\tau})-j(\boldsymbol{\sigma}) \geq 0
\end{aligned}
$$

for any $\boldsymbol{\tau} \in H(\operatorname{div}, \Omega)$, and altogether we arrive at the variational inequality of mixed form: find $[\mathbf{p}, \boldsymbol{\sigma}, u] \in L^{2}\left(\Omega, \mathbb{R}^{2}\right) \times$ $H(\operatorname{div}, \Omega) \times L^{2}(\Omega)$, such that for all $[\mathbf{q}, \boldsymbol{\tau}, v] \in L^{2}\left(\Omega, \mathbb{R}^{2}\right) \times$ $H(\operatorname{div}, \Omega) \times L^{2}(\Omega)$,

$$
\begin{gathered}
(\mathbf{a}(\cdot, \mathbf{p}), \mathbf{q})-(\boldsymbol{\sigma}, \mathbf{q})=0, \\
(\mathbf{p}, \boldsymbol{\tau}-\boldsymbol{\sigma})+(u, \operatorname{div}(\boldsymbol{\tau}-\boldsymbol{\sigma}))+j(\boldsymbol{\tau})-j(\boldsymbol{\sigma}) \geq 0, \\
-(v, \operatorname{div} \boldsymbol{\sigma}-u)=(f, v) .
\end{gathered}
$$

\section{Differential Mixed Variational Inequalities and Their Stability}

Motivated by the non-smooth boundary value problems and their variational formulation in the previous sections, we deal in this section with a general class of differential mixed variational inequalities. As we will see that, with some changes of notation, all the concrete variational inequalities of mixed form of the previous sections can be subsumed in this class, when introducing some appropriate product spaces.

Since in our stability analysis, we permit perturbations in the non-smooth convex functionals and in the convex constraint set, we provide auxiliary results on epiconvergence and Mosco convergence. Using the monotonicity method of Browder and Minty, we can establish a general stability result under weak convergence assumptions.

4.1. The Setting of Differential Mixed Variational Inequalities Considered. Let $X, V$ be two real, separable Hilbert spaces that are endowed with norms $\|\cdot\|_{X},\|\cdot\|_{V}$, respectively, and with scalar products denoted by $\langle\cdot, \cdot\rangle,(\cdot, \cdot)$, respectively. Further let there be given $T>0$, a convex closed subset $K \subset V$, a convex, lower semicontinuous proper functional $\phi: V \rightarrow \mathbb{R} \cup+\infty$, maps $f:[0, T] \times X \times V \rightarrow X$, and $g:[0, T] \times X \times V \rightarrow V$, and some fixed $x_{0} \in X$, then we consider the following problem: find a $X$-valued function $x$ and a $V$-valued function $u$ both defined on $[0, T]$ that satisfy for a.a. (almost all) $t \in[0, T]$

$$
\text { DMVI: }\left(f, g, K, \phi ; x_{0}\right)\left\{\begin{array}{l}
\dot{x}(t)=f(t, x(t), u(t)) \\
u(t) \in \Sigma(K, \phi, g(t, x(t), \cdot))
\end{array}\right.
$$

complemented by the initial condition $x(0)=x_{0}$. Here $\dot{x}(t)$ denotes the time derivative of $x(t) . \Sigma(K, \phi, g(t, x(t), \cdot))$ stands 
for the solution set of the mixed variational inequality defined by $K, \phi$, and $g(t, x(t), \cdot)$; that is, $u(t)$ has to satisfy

$$
\begin{array}{r}
u(t) \in K, \\
(g(t, x(t), u(t)), z-u(t))+\phi(z)-\phi(u(t)) \geq 0, \\
\forall z \in K .
\end{array}
$$

To give a precise meaning to a DMVI we have to introduce appropriate function spaces and impose some hypotheses on the data.

The fixed finite time interval $[0, T]$ gives rise to the Hilbert space $L^{2}(0, T ; V)$ endowed with the scalar product

$$
[u, v]:=\int_{0}^{T}(u(t), v(t)) d t .
$$

As in $[8,9]$ we consider weak solutions of the differential equation in a DMVI in the sense of Caratheodory. In particular, the $X$-valued function $x$ has to be absolutely continuous with derivative $\dot{x}(t)$ defined almost everywhere. Moreover to define the initial condition, the "trace" $x(0)$ is needed. Therefore (see [23, Theorem 1, page 473]) we are led to the function space

$$
\mathscr{X}(0, T ; X):=\left\{x \mid x \in L^{2}(0, T ; X), \dot{x}(t) \in L^{2}(0, T ; X)\right\},
$$

a Hilbert space endowed with the scalar product $[x, y]+$ $[\dot{x}, \dot{y}]$. Note that $\mathscr{X}(0, T ; X)$ is continuously and densely embedded in the space $C[0, T ; X]$ of $X$-valued continuous functions on $[0, T]$, where the latter space is equipped with the norm of uniform convergence.

We assume that the map $g$ satisfies the following growth condition: there exist $g_{0} \in L^{\infty}(0, T)$, and $g^{0} \in L^{2}(0, T)$ such that for all $t \in(0, T)$, for all $(x, u) \in X \times V$ there holds

$$
\|g(t, x, u)\|_{V} \leq g_{0}(t)\left(\|x\|_{X}+\|u\|_{V}\right)+g^{0}(t) .
$$

Hence the Nemytskii operator $G$ that acts from $L^{2}(0$, $T ; X) \times L^{2}(0, T ; V)$ to $L^{2}(0, T ; V)$ derives from $g$ by

$$
G(x, u)(t):=g(t, x(t), u(t)), \quad t \in(0, T) .
$$

For the time-independent functional $\phi$, we simply require that the functional $\Phi$ given by

$$
\Phi(u):=\int_{0}^{T} \phi(u(t)) d t, \quad u \in L^{2}(0, T ; V)
$$

is real valued on $L^{2}(0, T ; V)$.

Then it makes sense to introduce the closed convex subset

$$
\begin{aligned}
\mathscr{K} & :=L^{2}(0, T ; K) \\
& :=\left\{w \in L^{2}(0, T ; V) \mid w(t) \in K, \forall \text { a.a. } t \in(0, T)\right\}
\end{aligned}
$$

and replace the previous pointwise formulation of the mixed variational inequality in a DMVI by its integrated counterpart,

$$
u \in \mathscr{K}, \quad[G(x, u), v-u]+\Phi(v)-\Phi(u) \geq 0, \quad \forall v \in \mathscr{K} .
$$

Clearly, it is sufficient to test the variational inequality with any dense subset of $\mathscr{K}$, for example, the $K$-valued continuous functions on $[0, T]$, as in [8, Section 2.1].

Concerning the map $f$, we assume the following growth condition similar to (31): there exist $h_{0} \in L^{\infty}(0, T)$, and $h^{0} \in$ $L^{2}(0, T)$ such that for all $t \in(0, T)$, for all $(x, u) \in X \times V$ there holds

$$
\|f(t, x, u)\|_{X} \leq h_{0}(t)\left(\|x\|_{X}+\|u\|_{V}\right)+h^{0}(t) .
$$

Hence the Nemitskii operator $F$ derived from $f$ by

$$
F(x, u)(t):=f(t, x(t), u(t)), \quad t \in(0, T)
$$

acts from $L^{2}(0, T ; X) \times L^{2}(0, T ; V)$ to $L^{2}(0, T ; X)$.

Using a standard device in dynamical systems (see, e.g., [24]), we can introduce the unknown $\tilde{x}:=(x, t)$ and write the previous DMVI as

$$
\begin{aligned}
\frac{d \tilde{x}}{d \tau}=\frac{d}{d \tau}\left(\begin{array}{c}
x \\
t
\end{array}\right) & =\tilde{f}(\tilde{x}, u):=\left(\begin{array}{c}
f(\tilde{x}(t), u(t)) \\
1
\end{array}\right), \\
u(t) & \in \Sigma(K, \phi, g(\tilde{x}(t), \cdot)),
\end{aligned}
$$

complemented by the initial condition $\tilde{x}(0)=\left(x_{0}, 0\right)$. Therefore in the following we can consider the autonomous problem without any loss of generality and drop in DMVI the dependence on $t$.

In what follows we study stability of differential mixed variational inequalities formulated as DMVI and admit perturbations $x_{0, n}$ of $x_{0}$ in the initial condition $x(0)=x_{0}, f^{n}, g^{n}$ of the maps $f: X \times V \rightarrow X, g: X \times V \rightarrow V, K_{n}$ of the convex closed subset $K \subset V$, and $\phi^{n}$ of the convex, lower semicontinuous proper functional $\phi: V \rightarrow \mathbb{R} \cup+\infty$. Suppose that $\left(x^{n}, u^{n}\right)$ solves (DMVI) $\left(f^{n}, g^{n}, K_{n}, \phi^{n} ; x_{0, n}\right)$, and assume that $\left(x^{n}, u^{n}\right) \rightarrow(x, u)$ with respect to an appropriate convergence for $X$-valued, respectively, $V$-valued functions on $[0, T]$. Then we seek conditions on $f^{n} \rightarrow f, g^{n} \rightarrow$ $g, K_{n} \rightarrow K, \phi^{n} \rightarrow \phi$, and $x_{0, n} \rightarrow x_{0}$ that guarantee that $(x, u)$ solves the limit problem (DMVI) $\left(f, g, K, \phi ; x_{0}\right)$. Such a stability result can be understood as a result of upper set convergence for the solution set of $(\mathrm{DMVI})\left(f, g, K, \phi ; x_{0}\right)$.

4.2. Preliminaries; Mosco Convergence of Sets; Epiconvergence of Functions. As the convergence of choice in variational analysis we employ Mosco set convergence for a sequence $\left\{K_{n}\right\}$ of closed convex subsets which is defined as follows. A sequence $\left\{K_{n}\right\}$ of closed convex nonvoid subsets of the Hilbert space $V$ is called Mosco convergent to a closed convex nonvoid subset $K$ of $V$, written as $K_{n} \stackrel{M}{\longrightarrow} K$, if and only if

$$
\sigma-\limsup _{n \rightarrow \infty} K_{n} \subset K \subset s-\liminf _{n \rightarrow \infty} K_{n}
$$

Here the prefix $\sigma$ and $\stackrel{\sigma}{\rightarrow}$ mean sequentially weak convergence in contrast to strong convergence denoted by the prefix $s$ and by $\stackrel{s}{\rightarrow}$. Further, lim sup, lim inf respectively are in the sense of Kuratowski upper, lower limits respectively of sequences of sets (see [25] for more information on Mosco 
convergence). Here we note that for the nonempty set $K$ the second inclusion provides $g_{n} \in K_{n}$, such that $g_{n} \stackrel{s}{\rightarrow} g$ for some $g \in K$. Clearly, $K_{n} \stackrel{M}{\longrightarrow} K$, if and only if $C_{n}:=K_{n}-g_{n} \stackrel{M}{\longrightarrow} C:=$ $K-g$, this simple translation argument shows that there is no loss of generality to assume when needed that $0 \in K_{n}, K$.

As a preliminary result we next show that Mosco convergence of convex closed sets $K_{n}$ inherits to Mosco convergence of the polars $K_{n}^{0}$ and to Mosco convergence of the associated sets $\mathscr{K}_{n}=L^{2}\left(0, T ; K_{n}\right)$, derived from $K_{n}$ similar to (34).

Lemma 2. Let $K_{n} \stackrel{M}{\longrightarrow} K$. Then (a) $K_{n}^{0} \stackrel{M}{\longrightarrow} K^{0}$; (b) $\mathscr{K}_{n} \stackrel{M}{\longrightarrow} \mathscr{K}$ in $L^{2}(0, T ; V)$.

Proof. To show (a) we verify that

(1) $\sigma-\limsup _{n \rightarrow \infty} K_{n}^{0} \subset K^{0}$.

Let $\zeta=\sigma-\lim _{n \rightarrow \infty} \zeta_{n}$ with $\zeta_{n} \in K_{n}^{0}$. Choose $z \in K$ arbitrarily. Then by assumption, there exists (eventually for a subsequence) $z_{n} \in K_{n}$ with $z=s-\lim _{n \rightarrow \infty} z_{n}$. By definition of polar, $\left(\zeta_{n}, z_{n}\right) \leq 1$, for all $n$, hence in the limit $(\zeta, z) \leq$ 1, for all $z \in K$ which gives $\zeta \in K^{0}$.

(2) $K^{0} \subset s-\liminf _{n \rightarrow \infty} K_{n}^{0}$.

By [25, Proposition 3.23], $s\left(K_{n}\right) \stackrel{M}{\longrightarrow} s(K)$, where

$$
s(K)(\zeta):=\sup _{z \in K}(\zeta, z), \quad(\forall \zeta \in V)
$$

is the support function of $K$. This means in particular that, for any $\zeta \in \operatorname{dom} s(K)$, there exists $\zeta_{n} \in \operatorname{dom} s\left(K_{n}\right)$, such that $\zeta=s-\lim \zeta_{n}$ and $s(K)(\zeta) \geq \lim \sup _{n \rightarrow \infty} s\left(K_{n}\right)\left(\zeta_{n}\right)$

Now let $\zeta \in K^{0}$. Then $s(K)(\zeta) \leq 1$, and hence $\zeta \in$ $\operatorname{dom} s(K)$. For the previous sequences $\left\{\zeta_{n}\right\},\left\{s_{n}:=s\left(K_{n}\right)\left(\zeta_{n}\right)\right\}$, we obtain $\lim \sup _{n} s_{n} \leq 1$. If for a subsequence $s_{n_{k}} \leq 1$ holds, then $\zeta_{n_{k}} \in K_{n_{k}}^{0}$ and the argument is complete. Otherwise, for almost all $n$ we have $s_{n}>1$. But then $\lim s_{n}=1, \widetilde{\zeta}_{n}:=s_{n}^{-1} \zeta_{n} \in$ $K_{n}^{0}$, and $s-\lim _{n} \widetilde{\zeta}_{n}=\zeta$; the proof of part (a) terminates.

To show (b) we verify that

(1) $\sigma-\lim \sup _{n \rightarrow \infty} L^{2}\left(0, T ; K_{n}\right) \subset L^{2}(0, T ; K)$.

Let $w=\sigma-\lim _{n \rightarrow \infty} w_{n}$ with $w_{n} \in L^{2}\left(0, T ; K_{n}\right)$. By the bipolar theorem $\left(K^{00}=K\right.$, assume here without loss of generality that $0 \in K)$ it is enough to show that for all $\zeta \in K^{0}$, for a.a. $t \in(0, T)$ there holds $(w(t), \zeta) \leq 1$.

Assume not. Then there exist $\widetilde{\zeta} \in K^{0}, A \subset(0,1)$ with measure $|A|>0$, such that $(w(t), \widetilde{\zeta})>1$ on $A$. Define $v=$ $(1 /|A|) \chi_{A} \widetilde{\zeta}$ (with $\chi_{A}$ denoting the characteristic function of $A)$. By part (a), there exist $\widetilde{\zeta}_{n} \in K_{n}^{0}$, such that $s-\lim _{n} \widetilde{\zeta}_{n}=$ $\widetilde{\zeta}$; hence for $v_{n}=(1 /|A|) \chi_{A} \widetilde{\zeta}_{n}$, we have $s-\lim _{n} v_{n}=v$ in $L^{2}(0, T ; V)$. By construction,

$$
\left[w_{n}, v_{n}\right]=\frac{1}{|A|} \int_{A}\left(w_{n}(t), \widetilde{\zeta}_{n}\right) d t \leq 1
$$

Thus we arrive at the limit at

$$
1 \geq[w, v]=\frac{1}{|A|} \int_{A}(w(t), \tilde{\zeta}) d t>1,
$$

a contradiction proving the claim.

(2) $\mathscr{K} \subset s-\liminf _{n \rightarrow \infty} \mathscr{K}_{n}$.
It is enough to verify the claim for a dense subset of $\mathscr{K}$; this follows from a diagonal sequence argument; see also [26, Lemma 2.6] for a similar reasoning. Here we use the wellknown fact from Bochner-Lebesgue integration theory that the set $\mathcal{S}(0, T ; V)$ of simple $V$-valued functions on $(0, T)$ is dense in $L^{2}(0, T ; V)$. This extends to density of $\mathcal{S}(0, T ; V) \cap \mathscr{K}$, the $K$-valued simple functions on $(0, T)$, in $\mathscr{K}$. This can be seen by taking averages or mean value approximations; see [26] for approximations on a multidimensional integration domain instead of the interval $(0, T)$.

Thus let $w$ be a $K$-valued simple function on $(0, T)$; that is, $w=\sum_{j \in J} \chi_{A_{j}} z_{j}$, where $J$ is finite, $z_{j} \in K$, and $A_{j} \subset(0, T)$ are pairwise disjoint with measure $\left|A_{j}\right|>0$ and $\cup_{j \in J} A_{j}=(0, T)$. Since $K_{n} \stackrel{M}{\longrightarrow} K$, there exist $z_{j, n} \in K_{n}$, such that for all $j \in$ $J, z_{j, n} \rightarrow z_{j}(n \rightarrow \infty)$. Hence $w_{n}:=\sum_{j \in J} \chi_{A_{j}} z_{j, n}$ lies in $\mathscr{K}_{n}$, and $w=s-\lim _{n \rightarrow \infty} w_{n}$ in $L^{2}(0, T ; V)$ follows.

Let us note that part (b) of the preceding lemma is of intrinsic interest for time-dependent variational inequalities. An analogous implication (b) (1). was already shown in [26] in the more general context of probability spaces instead of the interval $(0, T)$, however for the restricted class of translated convex closed cones.

Since the epigraph epi $\phi:=\{(r, w): r \geq \phi(w)\}$ of a convex, lower semicontinuous (lsc) proper function $\phi$ : $V \rightarrow \mathbb{R} \cup+\infty$ is nonempty, closed, and convex in $\mathbb{R} \times$ $V$, Mosco convergence applies to such sets. This is known as epiconvergence (see e.g., [25]). Thus a sequence $\left\{\phi_{n}\right\}$ of convex lsc proper functions on the Hilbert space $V$ is called epiconvergent to a convex lsc proper function $\phi$ on $V$, written $\phi_{n} \stackrel{\text { epi }}{\longrightarrow} \phi$, if and only if

(1) $w=\sigma-\lim _{n \rightarrow \infty} w_{n} \Longrightarrow \phi(w) \leq \liminf _{n \rightarrow \infty} \phi_{n}\left(w_{n}\right)$,

(2) for all $w$ with $\phi(w)<+\infty \exists\left\{w_{n}\right\}_{n \in \mathbb{N}}$,

$$
w=s-\lim _{n \rightarrow \infty} w_{n}, \quad \phi(w) \geq \limsup _{n \rightarrow \infty} \phi_{n}\left(w_{n}\right)
$$

As a further preliminary result we next show that epiconvergence of convex lsc functions $\phi_{n}$ inherits to epiconvergence of the associated functionals $\Phi_{n}$, derived from $\phi_{n}$ similar to (33).

Lemma 3. Let the convex lsc proper functionals $\phi_{n}$ epiconverge to a convex lsc proper functional $\phi$ on $V$. Suppose that the functionals $\phi_{n}$ are equi-lower bounded in the sense that there exist $c_{o} \in \mathbb{R}, w_{0} \in V$, such that

$$
\phi_{n}(w) \geq c_{0}+\left(w_{0}, w\right), \quad \forall n \in \mathbb{N}, w \in V
$$

Then the associated functionals $\Phi_{n}$ epiconverge to $\Phi$ on $L^{2}(0, T ; V)$.

Proof. To show (1) let $v=\sigma-\lim _{n \rightarrow \infty} v_{n}$ in $L^{2}(0, T ; V)$. Hence $v(t)=\sigma-\lim _{n \rightarrow \infty} v_{n}(t)$ in $V$ for almost all $t \in(0, T)$. Now by 
equi-lower boundedness, conclude from the lemma of Fatou using the nonnegativity of the integrand that

$$
\begin{aligned}
& \liminf _{n \rightarrow \infty}\left\{\Phi_{n}\left(v_{n}\right)-\int_{0}^{T}\left[c_{0}+\left(w_{0}, v_{n}(t)\right)\right] d t\right\} \\
& \quad=\liminf _{n \rightarrow \infty} \int_{0}^{T}\left[\phi_{n}\left(v_{n}(t)\right)-c_{0}-\left(w_{0}, v_{n}(t)\right)\right] d t \\
& \quad \geq \int_{0}^{T} \liminf _{n \rightarrow \infty}\left[\phi_{n}\left(v_{n}(t)\right)-c_{0}-\left(w_{0}, v_{n}(t)\right)\right] d t \\
& \quad \geq \Phi(v)-\int_{0}^{T}\left[c_{0}+\left(w_{0}, v(t)\right)\right] d t
\end{aligned}
$$

To show (2) let $u \in L^{2}(0, T ; V)$ with $\Phi(u)<\infty$. Hence $\rho(t):=\phi(u(t))$ is real valued for almost all $t \in(0, T)$ and $[\rho, u] \in L^{2}(0, T$; epi $\phi)$. Now in virtue of the previous Lemma 2 , part (b), there exists a sequence $\left\{\left[\rho_{n}, u_{n}\right]\right\}$ strongly convergent to $[\rho, u] \in L^{2}(0, T ; \mathbb{R} \times V)$. Hence $u_{n} \rightarrow u \in$ $L^{2}(0, T ; V), u_{n}(t) \rightarrow u(t) \in V$ for almost all $t$, and $\rho_{n} \rightarrow \rho \in$ $L^{2}(0, T)$. Therefore conclude from the lemma of Fatou using the nonpositivity of the integrand that

$$
\begin{aligned}
& \limsup _{n \rightarrow \infty}\left\{\Phi_{n}\left(u_{n}\right)-\int_{0}^{T} \rho(t) d t\right\} \\
& \quad=\limsup _{n \rightarrow \infty} \int_{0}^{T}\left[\phi_{n}\left(u_{n}(t)\right)-\rho_{n}(t)\right] d t \\
& \leq \int_{0}^{T} \limsup _{n \rightarrow \infty}\left[\phi_{n}\left(u_{n}(t)\right)-\rho_{n}(t)\right] d t \\
& \leq \Phi(u)-\int_{0}^{T} \rho(t) d t .
\end{aligned}
$$

By combination of the previous lemmata we obtain the following auxiliary result.

Lemma 4. Let $K_{n} \stackrel{M}{\longrightarrow} K$ in $V$. Let the convex lsc proper functionals $\phi_{n}: K_{n} \rightarrow \mathbb{R}$ epiconverge to $\phi: K \rightarrow \mathbb{R}$ on $V$. Suppose that the functionals $\phi_{n}$ are equi-lower bounded in the sense that there exist $c_{o} \in \mathbb{R}, w_{0} \in V$, such that

$$
\phi_{n}\left(w_{n}\right) \geq c_{0}+\left(w_{0}, w_{n}\right), \quad \forall n \in \mathbb{N}, w_{n} \in K_{n} .
$$

Then the associated functionals $\Phi_{n}: L^{2}\left(0, T ; K_{n}\right) \rightarrow \mathbb{R}$ epiconverge to $\Phi: L^{2}(0, T ; K) \rightarrow \mathbb{R}$ in $L^{2}(0, T ; V)$.

The details of the proof are omitted.

As a further tool in our stability analysis we recall from [17] the following technical result.

Lemma 5. Let $H$ be a separable Hilbert space, and let $T>0$ be fixed. Then for any sequence $\left\{z_{n}\right\}_{n \in \mathbb{N}}$ converging to some $z$ in $L^{1}(0, T ; H)$ there exists a subsequence $\left\{z_{n_{k}}\right\}_{k \in \mathbb{N}}$, such that for some set $N$ of zero measure, $z_{n_{k}}(t) \stackrel{s}{\rightarrow} z(t)$ for all $t \in[0, T] \backslash N$.
4.3. The Stability Result. Before stating the result, some remarks are in order. In view of the existence theory of variational inequalities in infinite dimensional spaces (see, e.g., [15]) the best one can hope for is weak convergence of the perturbations $u^{n}$ in the general case of nonunique solutions of the underlying variational inequalities in (DMVI). Weak convergence can, namely, be readily derived from a posteriori estimates. However, continuity of a nonlinear map (here $g, f$ ) with respect to weak convergence is a hard requirement. To circumvent these weak convergence difficulties we apply the monotonicity method of Browder and Minty. Then as we shall see below, a stability condition on the maps $g^{n}$ with respect to the basic Hilbert space norm suffices.

These weak convergence difficulties also affect $f^{n}$. Therefore we have to impose a generally strong stability condition on the nonlinear maps $f^{n}$. In the situation of linear operators this condition can be drastically simplified to a stability condition with respect to convergence in the operator norm, see [18, Theorem 4.1] in the case $\phi=\phi^{n}=0$.

On the other hand, stronger assumptions on $g^{n}$, like uniform monotonicity, imply that the solution sets $\Sigma\left(K_{n}, \phi^{n}, G\left(x^{n}, \cdot\right)\right)$ are single valued. Uniform monotonicity with respect to $n$ moreover entails that the sequence $u^{n}$ strongly converges. Then the stability assumption for $f^{n}$ can be relaxed.

Since our stability assumptions pertain the given maps $g^{n}, g$, not the derived maps $G^{n}, G$, we have a delicate interplay between the pointwise almost everywhere formulation and the integrated formulation of the variational inequality in the perturbed DMVI and in the limit DMVI.

We need the following hypotheses on the convergence of $\left(f^{n}, g^{n}\right)$ to $(f, g)$.

(H1) Let $z_{n} \stackrel{s}{\rightarrow} z$ in $X$ and $w_{n} \stackrel{\sigma}{\rightarrow} w$ in $V$. Moreover, let $f^{n}\left(z_{n}, w_{n}\right) \stackrel{s}{\rightarrow} p$ in $X$. Then $p=f(z, w)$.

(H2) All maps $g^{n}(z, \cdot)$ for any $z \in X$ are monotone. If $z_{n} \stackrel{s}{\rightarrow} z, w_{n} \stackrel{s}{\rightarrow} w$ in $X$, and $V$ respectively, then $g^{n}\left(z_{n}, w_{n}\right) \stackrel{s}{\rightarrow} g(z, w)$ in $V . g$ is hemicontinuous in the sense that for any $z \in X ; v, w \in V$, the realvalued function $r \in \mathbb{R} \mapsto(g(z, v+r w), r w)$ is lower semicontinuous.

Now we can state the following stability result.

Theorem 6. Let $\left(x^{n}, u^{n}\right)$ solve (DMVI) $\left(f^{n}, g^{n}, K_{n}, \phi^{n} ; x_{0, n}\right)$. Suppose that $f^{n}, f, g^{n}$, and $g$, respectively, satisfy (H1), (H2), respectively. Let $x_{0, n} \stackrel{s}{\rightarrow} x_{0}$. Let the convex closed nonvoid sets $K_{n}$ Mosco-converge to $K$ in $V$, and let the convex lsc proper functions $\phi^{n}: K_{n} \rightarrow \mathbb{R}$ epiconverge to $\phi: K \rightarrow \mathbb{R}$ on $V$. Suppose that the functions $\phi^{n}$ are equi-lower bounded in the sense of (47). Assume that $x^{n} \stackrel{s}{\rightarrow} x$ in $\mathscr{X}(0, T ; X)$ and that $u^{n} \in L^{2}(0, T ; V)$ converges weakly to $u$ pointwise in $V$ for a.a. $t \in(0, T)$ with $\left\|u^{n}(t)\right\|_{V} \leq m(t)$, for all a.a. $t \in(0, T)$ for some $m \in L^{2}(0, T)$. Then $(x, u)$ is a solution to (DMVI) $\left(f, g, K, \phi ; x_{0}\right)$.

Proof. The proof consists of three parts.

(1) Feasibility: $u \in \mathscr{K}, x(0)=x_{0}$. 
First we observe that, for any $w \in L^{2}(0, T ; V)$, in virtue of Lebesgue's theorem of dominated convergence,

$$
\left[u^{n}, w\right]=\int_{0}^{T}\left(u^{n}(t), w(t)\right) d t \longrightarrow[u, w] .
$$

Thus $u^{n} \stackrel{\sigma}{\rightarrow} u$ and $u \in L^{2}(0, T ; V)$. Moreover directly by Mosco convergence of $\left\{K_{n}\right\}$ or invoking Lemma 2 (b), $u \in \mathscr{K}$ follows.

Since by continuous embedding $x^{n} \stackrel{s}{\rightarrow} x$ in $C[0, T ; X]$, we conclude that $x^{n}(0)=x_{0, n} \stackrel{s}{\rightarrow} x(0)=x_{0}$.

(2) $u$ solves the variational inequality in (DMVI) $(f, g, K$, $\left.\phi ; x_{0}\right)$ :

$$
u(t) \in \Sigma(K, \phi, g(x(t), \cdot)), \quad \forall \text { a.a. } t \in(0, T) .
$$

Fix an arbitrary $w \in \mathscr{K}$. Then by Lemma 4 , there exist $w^{n} \in \mathscr{K}_{n}$, such that $w^{n} \stackrel{s}{\rightarrow} w$ in $L^{2}(0, T ; V)$ and $\lim \sup _{n} \Phi^{n}\left(w^{n}\right) \leq \Phi(w)$. Moreover, by extracting eventually a subsequence, we have by Lemma 5 that also $w^{n}(t)$ strongly converges to $w(t)$ for a.a. $t \in(0, T)$. For any measureable set $A \subset(0, T)$ we can define $w_{A}^{n} \in L^{2}(0, T ; V)$ by $w_{A}^{n}=w^{n}$ on $A$, $w_{A}^{n}=u^{n}$ on $(0, T) \backslash A$. Hence $w_{A}^{n} \in \mathscr{K}_{n}$ and by construction it follows:

$$
\begin{gathered}
\int_{A}\left(g^{n}\left(x^{n}(t), u^{n}(t)\right), w^{n}(t)-u^{n}(t)\right) \\
+\phi^{n}\left(w^{n}(t)\right)-\phi^{n}\left(u^{n}(t)\right) d t \geq 0 .
\end{gathered}
$$

Hence a contradiction argument shows that we have pointwise for a.a. $t \in(0, T)$,

$$
\begin{gathered}
\left(g^{n}\left(x^{n}(t), u^{n}(t)\right), w^{n}(t)-u^{n}(t)\right) \\
+\phi^{n}\left(w^{n}(t)\right)-\phi^{n}\left(u^{n}(t)\right) \geq 0 .
\end{gathered}
$$

By (H2), monotonicity entails pointwise for a.a. $t \in(0, T)$ as follows:

$$
\begin{aligned}
& \left(g^{n}\left(x^{n}(t), w^{n}(t)\right), u^{n}(t)-w^{n}(t)\right) \\
& \quad \leq \phi^{n}\left(w^{n}(t)\right)-\phi^{n}\left(u^{n}(t)\right) .
\end{aligned}
$$

Again by (H2), the growth condtion (31), and by epiconvergence, we arrive taking the lim sup at

$$
\begin{aligned}
{[G(x, w), u-w] } & =\int_{0}^{T}(g(x(t), w(t)), u(t)-w(t)) d t \\
& \leq \Phi(w)-\Phi(u), \quad \forall w \in \mathscr{K} .
\end{aligned}
$$

Hence by a well-known argument in monotone operator theory (see, e.g., [16]) we obtain that $u \in \mathscr{K}$ satisfies the variational inequality

$$
[G(x, u), w-u]+\Phi(w)-\Phi(u) \geq 0, \quad \forall w \in \mathscr{K},
$$

provided that the map $G(x, \cdot)$ is hemicontinuous. But this follows from the hemicontinuity assumption in (H2) for $g(x, \cdot)$ as follows. To show for any $y \in L^{2}(0, T ; X) ; v, w \in$ $L^{2}(0, T ; V)$,

$$
\liminf _{r \rightarrow r_{0}}[G(y, v+r w), r w] \geq\left[G\left(y, v+r_{0} w\right), r_{0} w\right],
$$

subtract

$$
\lim _{r \rightarrow r_{0}}[G(y, v), r w]=\left[G(y, v), r_{0} w\right],
$$

and apply Fatou's lemma to the nonnegative integrand to conclude that

$$
\begin{gathered}
\liminf _{r \rightarrow r_{0}} \int_{0}^{T}(g(y(t), v(t)+r w(t)), r w(t)) d t \\
\quad \geq \int_{0}^{T} \liminf _{r \rightarrow r_{0}}(g(y(t), v(t)+r w(t)), r w(t)) d t \\
\quad \geq \int_{0}^{T}\left(g\left(y(t), v(t)+r_{0} w(t)\right), r_{0} w(t)\right) d t .
\end{gathered}
$$

(3) $(x, u)$ solves the limit (DMVI) $\left(f, g, K, \phi ; x_{0}\right)$.

By Lemma 5 applied to $\left\{x^{n}\right\}$ and $\left\{\dot{x}^{n}\right\}$, we can extract a subsequence, such that $x^{n}(t) \rightarrow x(t)$ and $\dot{x}^{n}(t) \rightarrow \dot{x}(t)$ strongly in $X$ pointwise for all $t \in(0, T) \backslash N_{0}$, where $N_{0}$ is a null set. Fix $t \in(0, T) \backslash N_{0}$. Then by assumption, for all $n \in \mathbb{N}$ we have $\dot{x}^{n}(t)=f^{n}\left(x^{n}(t), u^{n}(t)\right)$. Then in virtue of (H1), $\dot{x}(t)=f(x(t), u(t))$ follows, and $(x, u)$ solves the $(\mathrm{DMVI})\left(f, g, K, \phi ; x_{0}\right)$.

When the DMVI has a separable structure, the hypotheses (H1) and (H2) can be expressed more explicitly. Similar to [9], we have

$$
\begin{gathered}
f(z, w)=f_{1}(z)+B(z) w, \\
f_{n}(z, w)=f_{1, n}(z)+B_{n}(z) w
\end{gathered}
$$

with linear operators $B, B_{n} \in \mathscr{L}(X, \mathscr{L}(V, X))$, then $B_{n} \rightarrow B$ in the operator norm along with $f_{1, n}\left(z_{n}\right) \stackrel{s}{\rightarrow} f_{1}(z)$ for $z_{n} \stackrel{s}{\rightarrow} z$ implies (H1).

More simply, also with [9], let

$$
\begin{gathered}
g(z, w)=g_{1}(z)+g_{2}(w), \\
g_{n}(z, w)=g_{1, n}(z)+g_{2, n}(w) .
\end{gathered}
$$

Then (H2) is satisfied, if the $g_{2, n}$ are monotone, $g_{2}$ is hemicontinuous, $g_{1, n}\left(z_{n}\right) \stackrel{s}{\rightarrow} g_{1}(z)$ for $z_{n} \stackrel{s}{\rightarrow} z$, and $g_{2, n}\left(w_{n}\right) \stackrel{s}{\rightarrow}$ $g_{2}(w)$ for $w_{n} \stackrel{s}{\rightarrow} w$.

In the variational inequalities of mixed form (see in particular (13)), we find this separable structure. Here in particular we have for $y=[p, \sigma]$,

$$
g_{2}(y)=\left(\begin{array}{cc}
\mathscr{A}(\cdot) & -C \\
C^{*} & 0
\end{array}\right)-\left(\begin{array}{l}
f_{0} \\
g_{0}
\end{array}\right)
$$

with fixed $f_{0}, g_{0}$, and an appropriate linear operator $C$ with its adjoint $C^{*}$. Then $g_{2}$ inherits monotonicity from the nonlinear operator $\mathscr{A}$.

We refrain from deriving a stability result for stationary problems from Theorem 6. Instead we can refer to [27, Theorem 3] for a much stronger result. 


\section{Some Concluding Remarks}

Let us first shortly remark on possible extensions and limitations of the previous stability result. The monotonicity method extends easily to set-valued operators. Also, monotonicity can be replaced by the more general, however more abstract notion of (order-) pseudomonotonicity.

Here motivated by the considered non-smooth boundary value problems we discussed differential variational inequalities in a Hilbert space framework. Let us point out that differential variational inequalities and their stability using Mosco convergence can be investigated in more general reflexive Banach spaces, but not beyond [28].

Finally let us give an outlook of some open directions of research. In this paper we confined the three-field modelling to a simplified scalar model of nonsmooth contact in continuum mechanics. An extension of the three-field duality approach to such non-smooth boundary value problems will involve the tensor fields of continuum mechanics. In particular, the basic Green's formula (5) has then to be replaced by the more general "integration by parts" formula $[29,(3.46)]$.

In this paper, we did not touch the issue of existence of solutions to DMVI. As shown by Pang and Stewart [8] and Li et al. respectively, [9], existence of solutions to DMVI can be obtained from the theory of multivalued differential equations [30] and the theory of the more general differential inclusions [31]. However, compactness assumptions as used in $[8,9]$ are too strong in infinite dimensions; moreover the feasible set $K$ defined by the unilateral boundary conditions is a convex cone, but not compact. For an existence result for linear differential variational inequalities based on maximal monotone operator theory, we can refer to [18].

When it comes to numerical approximation, note that Galerkin approximation with respect to space discretization leads in the case of higher than piecewise linear approximation; to a nonconforming approximation, that is, the approximating convex set $K_{h}$ is not a subset of the given convex $K$. In this situation Mosco convergence (and more refined Glowinski convergence) is instrumental to arrive at convergence of Galerkin approximation; see [7,32]. Thus our stability result can be seen as an important step towards convergence of semidiscretization methods that provide finite dimensional differential variational inequalities. Clearly a full space time discretization needs an additional analysis (see, e.g., [33]) and is beyond the scope of the present paper.

\section{References}

[1] G. N. Gatica, "An application of Babuška-Brezzi’s theory to a class of variational problems," Applicable Analysis, vol. 75, no. 3-4, pp. 297-303, 2000.

[2] G. N. Gatica, "Solvability and Galerkin approximations of a class of nonlinear operator equations," Zeitschrift für Analysis und ihre Anwendungen, vol. 21, no. 3, pp. 761-781, 2002.

[3] G. N. Gatica, N. Heuer, and S. Meddahi, "On the numerical analysis of nonlinear twofold saddle point problems," IMA Journal of Numerical Analysis, vol. 23, no. 2, pp. 301-330, 2003.
[4] I. Ekeland and R. Temam, Analyse Convexe et Problèmes Variationnels, Dunod, Gauthier-Villars, Paris, France, 1974.

[5] J. Haslinger, "Mixed formulation of elliptic variational inequalities and its approximation," Aplikace Matematiky, vol. 26, no. 6, pp. $462-475,1981$.

[6] J. Haslinger and J. Lovíšek, "Mixed variational formulation of unilateral problems," Commentationes Mathematicae Universitatis Carolinae, vol. 21, no. 2, pp. 231-246, 1980.

[7] I. Hlaváček, J. Haslinger, J. Nečas, and J. Lovíšek, Numerical Solution of Variational Inequalities, vol. 66 of Applied Mathematical Sciences, Springer, New York, NY, USA, 1988.

[8] J.-S. Pang and D. E. Stewart, "Differential variational inequalities," Mathematical Programming, vol. 113, no. 2, pp. 345-424, 2008.

[9] X.-S. Li, N.-J. Huang, and D. O’Regan, "Differential mixed variational inequalities in finite dimensional spaces," Nonlinear Analysis: Theory, Methods \& Applications, vol. 72, no. 9-10, pp. 3875-3886, 2010.

[10] D. Goeleven, D. Motreanu, and V. V. Motreanu, "On the stability of stationary solutions of first order evolution variational inequalities," Advances in Nonlinear Variational Inequalities, vol. 6, no. 1, pp. 1-30, 2003.

[11] J.-S. Pang and D. E. Stewart, "Solution dependence on initial conditions in differential variational inequalities," Mathematical Programming, vol. 116, no. 1-2, pp. 429-460, 2009.

[12] N. S. Papageorgiou, "A stability result for differential inclusions in Banach spaces," Journal of Mathematical Analysis and Applications, vol. 118, no. 1, pp. 232-246, 1986.

[13] N. S. Papageorgiou, "On parametric evolution inclusions of the subdifferential type with applications to optimal control problems," Transactions of the American Mathematical Society, vol. 347, no. 1, pp. 203-231, 1995.

[14] S. Hu and N. S. Papageorgiou, "Time-dependent subdifferential evolution inclusions and optimal control," Memoirs of the American Mathematical Society, vol. 133, no. 632, 81 pages, 1998.

[15] D. Kinderlehrer and G. Stampacchia, An Introduction to Variational Inequalities and Their Applications, vol. 88 of Pure and Applied Mathematics, Academic Press, New York, NY, USA, 1984.

[16] E. Zeidler, "Nonlinear Monotone Operators," in Nonlinear Functional Analysis and Its Applications, part 2, Springer, New York, NY, USA, 1990.

[17] J. Gwinner, "On differential variational inequalities and projected dynamical systems-equivalence and a stability result," Discrete and Continuous Dynamical Systems A, vol. 2007, pp. 467-476, 2007.

[18] J. Gwinner, "A note on linear differential variational inequalities in Hilbert space," in Modeling and Optimization, D. Hömberg and F. Tröltzsch, Eds., pp. 85-91, Springer, Heidelberg, Germany, 2013.

[19] H. S. Carslaw and J. C. Jaeger, Conduction of Heat in Solids, Clarendon, New York, NY, USA, 2nd edition, 1988.

[20] G. Duvaut and J.-L. Lions, Inequalities in Mechanics and Physics, Springer, Berlin, Germany, 1976.

[21] J. Nečas, Les Méthodes Directes en Théorie des Équations Elliptiques, Masson, Paris, France, 1967.

[22] V. Girault and P.-A. Raviart, Finite Element Approximation of the Navier-Stokes Equations, vol. 749 of Lecture Notes in Mathematics, Springer, Berlin, Germany, 1981.

[23] R. Dautray and J.-L. Lions, Mathematical Analysis and Numerical Methods for Science and Technology, vol. 5, Springer, Berlin, Germany, 1992. 
[24] M. Kunze, Non-Smooth Dynamical Systems, vol. 1744 of Lecture Notes in Mathematics, Springer, Berlin, Germany, 2000.

[25] H. Attouch, Variational Convergence for Functions and Operators, Applicable Mathematics Series, Pitman, Boston, Mass, USA, 1984.

[26] J. Gwinner, "A class of random variational inequalities and simple random unilateral boundary value problems-existence, discretization, finite element approximation," Stochastic Analysis and Applications, vol. 18, no. 6, pp. 967-993, 2000.

[27] J. Gwinner, "Stability of monotone variational inequalities with various applications," in Variational Inequalities and Network Equilibrium Problems, F. Giannessi and A. Maugeri, Eds., pp. 123-142, Plenum, New York, NY, USA, 1995.

[28] G. Beer and J. M. Borwein, "Mosco convergence and reflexivity," Proceedings of the American Mathematical Society, vol. 109, no. 2, pp. 427-436, 1990.

[29] F. Brezzi and M. Fortin, Mixed and Hybrid Finite Element Methods, vol. 15 of Springer Series in Computational Mathematics, Springer, New York, NY, USA, 1991.

[30] K. Deimling, Multivalued Differential Equations, vol. 1 of de Gruyter Series in Nonlinear Analysis and Applications, Walter de Gruyter, Berlin, Germany, 1992.

[31] J.-P. Aubin and A. Cellina, Differential Inclusions, vol. 264 of Grundlehren der Mathematischen Wissenschaften, Springer, Berlin, Germany, 1984.

[32] R. Glowinski, Numerical Methods for Nonlinear Variational Problems, Springer Series in Computational Physics, Springer, New York, NY, USA, 1984.

[33] C. Carstensen and J. Gwinner, "A theory of discretization for nonlinear evolution inequalities applied to parabolic Signorini problems," Annali di Matematica Pura ed Applicata, vol. 177, pp. 363-394, 1999. 


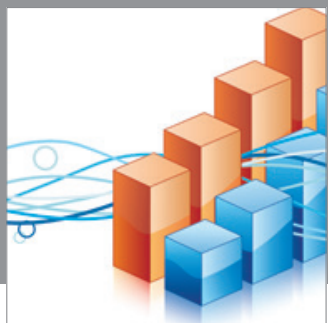

Advances in

Operations Research

mansans

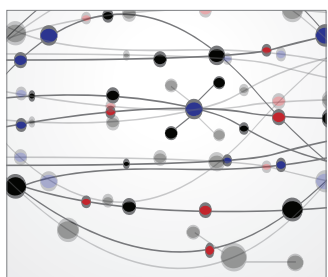

The Scientific World Journal
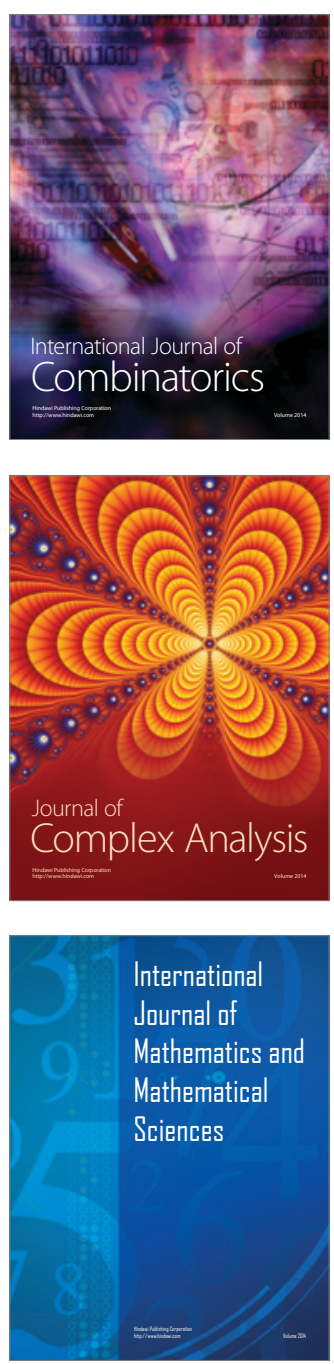
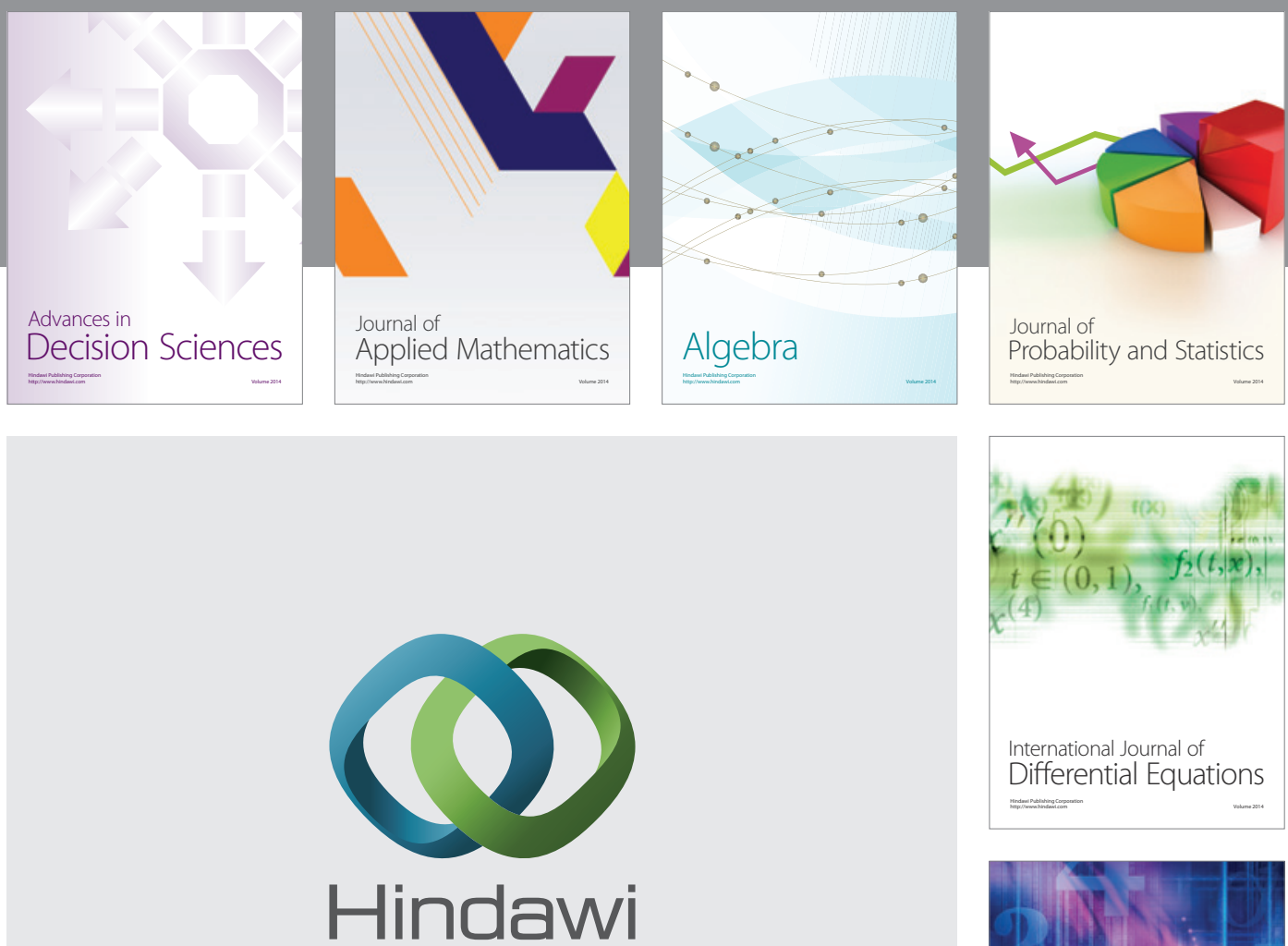

Submit your manuscripts at http://www.hindawi.com
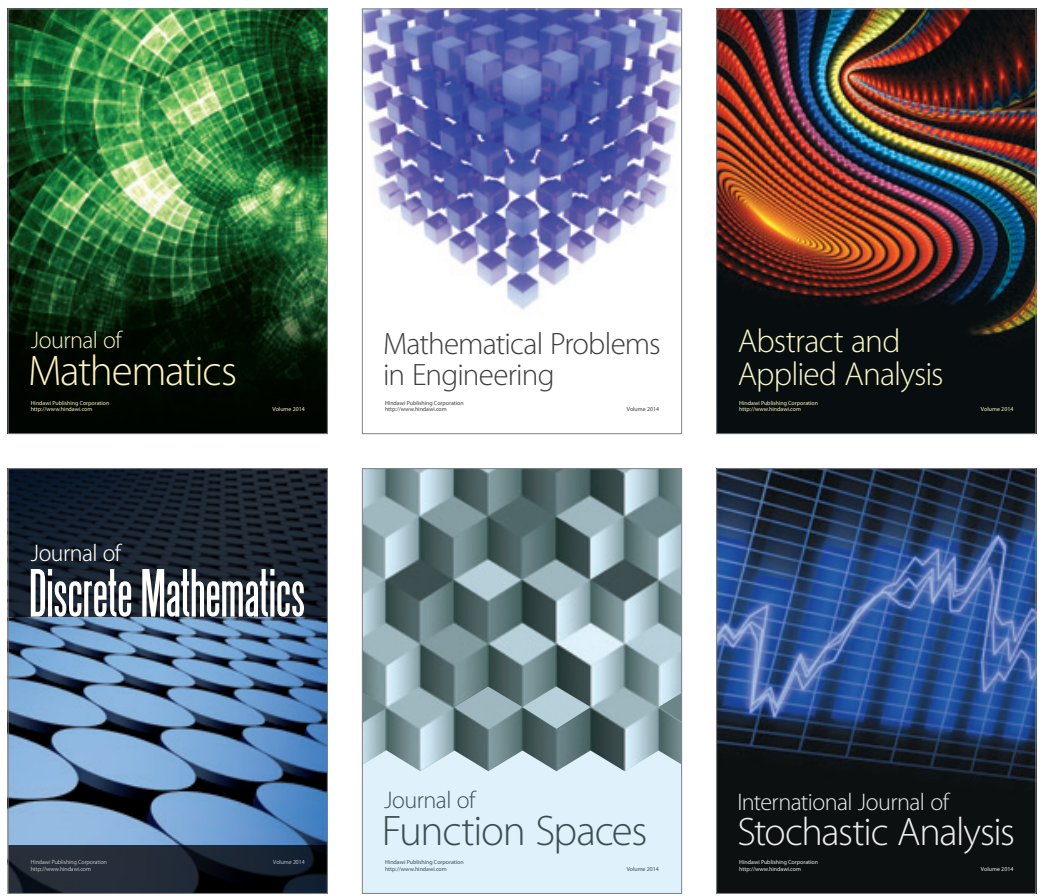

Journal of

Function Spaces

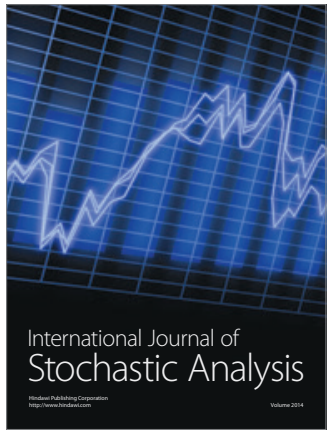

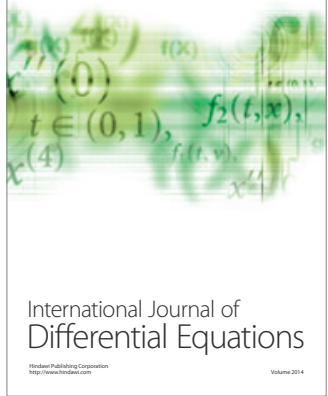
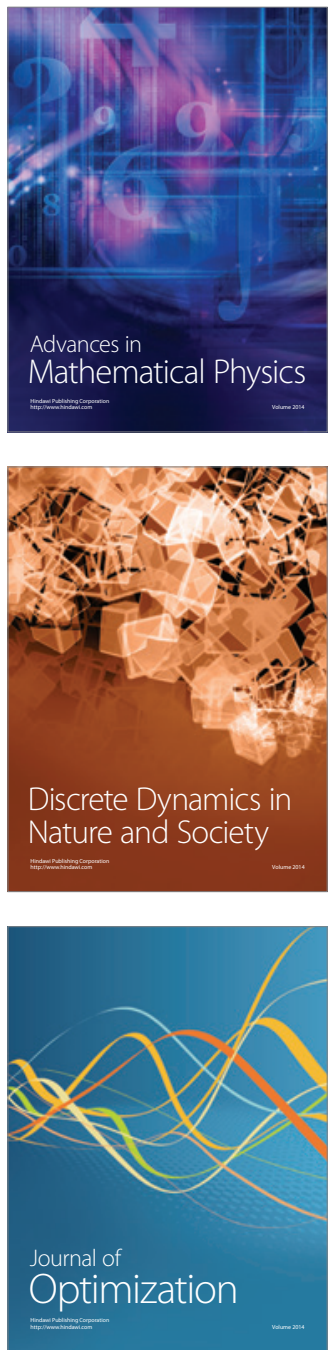